\title{
T221. Sensorimotor Induction of Auditory Misattribution in Psychosis is Linked to Neural Disconnectivity
}

Roy Salomon1, Pierre Progin2, Alessandra Griffa3, Giulio Rognini1, Kim Q. Do4, Philippe Conus2, Silvia Marchesotti1, Patric Hagmann3, Andrea Serino1, and Olaf Blanke5

1 Center for Neuroprosthetics, School of Life Sciences, Ecole Polytechnique Fédérale de Lausanne (EPFL), Brain Mind Institute, 2 Lausanne University Hospital, 3 Lausanne University Hospital (CHUV) and University of Lausanne, Ecole Polytechnique Fédérale de Lausanne (EPFL), 4 Center for Psychiatric Neuroscience, Lausanne University Hospital, 5 Center for Neuroprosthetics, School of Life Sciences, Ecole Polytechnique Fédérale de Lausanne (EPFL), Brain Mind Institute, University Hospital, Geneva

\section{Abstract the poster presented at the 2018 SOBP (Society of Biological Psychiatry) Annual Meeting in New York (USA)}

Background: Schneiderian first rank symptoms (FRS) are characterized by a diminished demarcation of self-other boundaries, causing misattribution of self-generated thoughts and actions to external sources. We have shown that introducing sensorimotor prediction error (SPE) by a robotic device in healthy subjects can induce a Feeling of a Presence (FoP) which is related to the FRS (Blanke et al. 2014). Here, we tested if SPE may induce auditory misattribution in psychotic patients and if this is related to neural connectivity in the temporoparietal cortex, insular cortex and fronto-parietal cortex (FoP Network).

Methods: Participants manipulated a haptic robotic system inducing a sensorimotor conflict while performing a self-other auditory discrimination task. 31 early psychotic patients (19 with and 12 without FRS) and 20 controls participated in the experiment. We measured accuracy (d') on auditory self-other discrimination task during sensorimotor conflict induction or control condition, functional connectivity magnitude in a priori FoP network, and calculated correlation between the two measures.

Results: Patients with FRS had reduced accuracy in auditory self-other discrimination when sensorimotor conflict was induced $(F(2,44)=6.68, p=.002)$. rsfMRI connectivity analysis indicated lower connectivity for these patients in regions of the FoP network compared to the non-first rank and control groups $(p=.015, p=.014)$. The level of functional connectivity in the FoP network correlated with the reduction of self-other discrimination in the FRS+ group $(r=-0.56, p=.03)$.

Conclusions: Experimental induction of SPE can cause self-other confusion in the auditory domain. This deficit in self-other discrimination was correlated to specifically reduced connectivity in the FoP network related to sensorimotor self-representation.

Supported By: National Center of Competence in Research (NCCR) "SYNAPSY - The Synaptic Bases of Mental Diseases" financed by the Swiss National Science Foundation (No. 51AU40_125759)

Keywords: Early Psychosis, Prediction Errors, First Rank Symptoms, Sensorimotor Processing, Robotic Stimulation 